\title{
On the Duration of Blackouts Due to Primary User Activity in Cognitive Networks
}

\author{
Jelena Mišić and Vojislav B. Mišić \\ Ryerson University \\ Toronto, ON, Canada M5B 2K3 \\ Email: \{jmisic, vmisic\}@ryerson.ca
}

\begin{abstract}
We derive probability distribution for the duration of total blackouts - periods in which no channel is idle - in the set of channels used by a cognitive network under random activity of independent primary users. We show that the mean value of blackout duration is very dependent on the number of channels in the network, and somewhat dependent on the activity factor (i.e., duty cycle) of primary user transmissions, with the impact of the latter being more pronounced in cases with smaller number of channels.
\end{abstract}

\section{INTRODUCTION}

Opportunistic or cognitive spectrum access (OSA) often relies on channel hopping as the basic mechanism to ensure communication [2], [1], [5]. Unpredictable activity patterns of licensed (primary) users may lead to a 'blackout' on one or more channels, during which the said channels are unavailable for use by the nodes in the cognitive (secondary) network.

- A partial blackout corresponds to the scenario where some channels are idle but others are busy, in which case the operation of the cognitive network may or may not be interrupted; still, the network may need to switch to an idle channel in order to continue its operation.

- A total blackout describs the scenario where primary users are active on all channels in the working set, in which case cognitive network must suspend its operation until at least one of the channels becomes idle (and, consequently, available for its use) again.

Obviously every blackout represents a disruption, which is why it is of interest to examine the conditions that lead to the blackout and its behavior. The results of this analysis can be used to design novel MAC protocols for cognitive networks [3], [6] and the associated mechanisms for network rendezvous [10], working channel selection [9], and cognitive network recovery [8], all of which should be resilient to disruptions from primary users or, at least, able to limit the impact from said disruptions to an amount close to the theoretical minimum.

In this paper, we examine the behavior of total blackouts under random primary user activity and analyze the probability distribution of blackout duration. We show that the mean value of blackout duration is mostly dependent on the number of channels in the network, and somewhat dependent on the activity factor (i.e., duty cycle) of primary user transmissions. The impact of the activity factor is shown to be more pronounced under smaller number of channels. These results are shown to hold for different probability distributions of active and idle channel times, including exponential, Erlang-2, and a mixed distribution with constant active and exponential idle times.

The paper is organized as follows. In Section II we use probabilistic analysis to model primary user activity and the conditions which lead to a blackout. Section III presents performance evaluation of the model, while Section IV concludes the work.

\section{Modeling THE BLACKOUTS}

Let the cognitive network use a total of $N$ channels, each of which hosts a primary user that exhibits random activity. Let us assume that the durations of active and idle times on each channel, $T_{a}$ and $T_{i}$, are independent, identically distributed (iid) random variables the probability density functions (pdf) $t_{a}(x)$ and $t_{i}(x)$, respectively. As active and idle times alternate, pdf of the total cycle time on the channel may be obtained as a convolution of pdf's of active and idle times, $t(x)=t_{i}(x) * t_{a}(x)$. Then, the probability that the channel is active or idle can be calculated as $p_{\text {on }}=\frac{\overline{T_{a}}}{\overline{T_{a}}+\overline{T_{i}}}$ and $p_{\text {off }}=1-p_{\text {on }}=\frac{\overline{T_{i}}}{\overline{T_{a}}+\overline{T_{i}}}$, respectively. Mean cycle time can, then, be calculated as

$$
\overline{T_{c y c}}=\overline{T_{a}}+\overline{T_{i}}
$$

while the corresponding mean number of idle and active channels are $\overline{N_{i}}=p_{\text {off }} N$ and $\overline{N_{a}}=p_{\text {on }} N$, respectively.

The problem of modeling the blackouts can be approached by making use of renewal theory as the major analytical tool. Namely, a random point process $\psi=\left\{t_{n}\right\}$ for which the interarrival times $\left\{X_{n}\right\}$ form an idd sequence is called a renewal process [4]. The beginning of new cycle is a renewal point at which a new probabilistic replica of the original renewal process starts. Furthermore, consider an arbitrary event occuring during the general cycle time $X_{i}$ at the time $t$ relative to the beginning of new cycle (renewal point). The period from onset of the cycle to $t$ is denoted as $X_{i,-}$ and is referred to as elapsed or deficit cycle time; similarly, the period from the time $t$ to the $X_{i,+}=X_{i}-t$ as residual or excess cycle time.

In the context of blackout periods on the channel set used by the cognitive network, cycle time is comprised of active and idle channel periods; the process that counts cycles of 


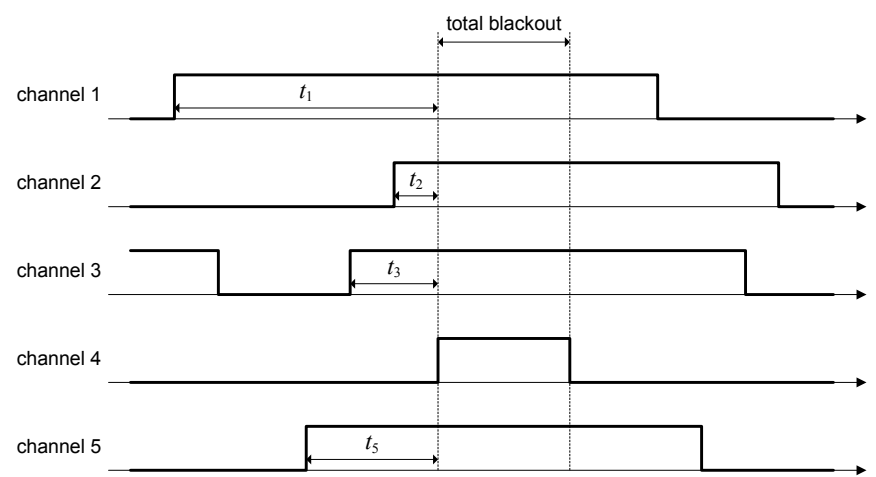

(a) Scenario 1: blackout ends 'on' the same channel (4) where it started.

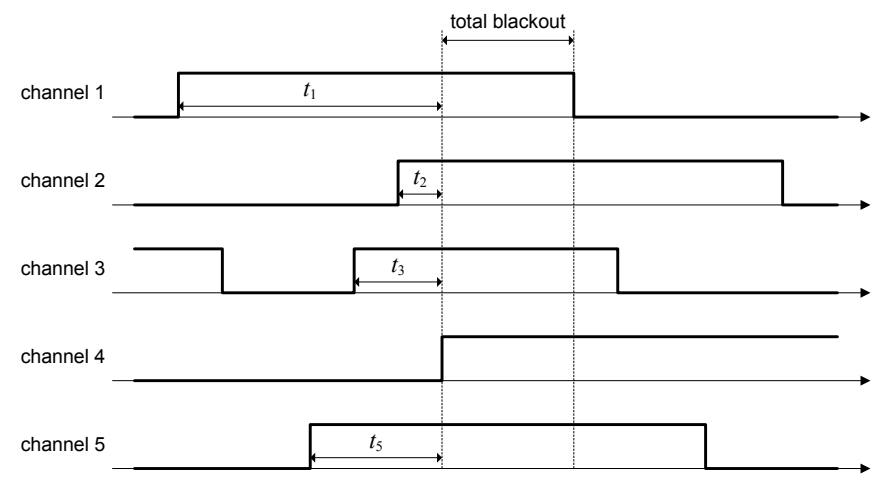

(b) Scenario 2: blackout begins on one channel (4) and ends 'on' a different one (1).

Fig. 1. Blackout timing.

primary activity starting from the onset of active period on some channel is a renewal process; finally, renewal points correspond to the beginning of active channel periods.

As noted above, a total blackout occurs when primary activity exists on all $N$ channels. In other words, assuming that primary users are already active on $N-1$ channels, blackout begins when the remaining channel becomes active and ends when primary activity ceases on any of the channels. There are two scenarios in which a total blackout may occur. In one of them, both the beginning and the end of the blackout are caused by the beginning and the end of primary activity on a single channel, as shown in Fig. 1(a). In the other, the channel on which the end of activity terminates the blackout may differ from the channel on which the beginning of primary activity has begun the blackout, as shown in Fig. 1(b).

Consider channels from Fig. 1, labeled as 1.. 5, and assume that all channels except channel $c h_{4}$ have already become active. Let the primary user activity begin on channel 4 , which begins the blackout period. Let the time between onset of activity at each channel and onset of activity at the channel 4 be denoted as $t_{1}, t_{2}, \ldots$, as shown in the diagrams.

Points $t_{1}, t_{2}, \ldots$ are random points in respective active periods of the channels which have already switched to active state (in this example, this includes all channels except channel 4). Therefore, the time between the beginning of the active period on channel $i$ and $t_{i}$ is elapsed active time, which we will denote with $T_{a,-}$. The time between the beginning of activity on channel 4 and the end of active period on channel $i \neq 4$ is residual active channel time, denoted with $T_{a,+}$. We will first calculate the elapsed cycle time $T_{a,-}$ using the known probability density function of channel active time $t_{a}(y)$. Since elapsed and residual cycle times are known to have identical probability distributions [4], this will also give us the residual channel time through $T_{a,+}=T_{a,-}$.

Let us denote the probability distribution function (PDF) of elapsed active channel time with $B(x)=P\left(T_{a,-} \leq x\right)$, while its pdf is $b(x)=\frac{d B(x)}{d x}$. Let us also define

$$
\begin{aligned}
T_{a}^{c}(x) & =1-B(x)=P\left(T_{a,-}>x\right) \\
& =\int_{y=x}^{\infty} t_{a}(y) d y .
\end{aligned}
$$

Then, the PDF of deficit channel active time $B(x)$ can be calculated as

$$
B(x)=\frac{1}{\overline{T_{a}}} \int_{0}^{x} T_{a}^{c}(y) d y
$$

and the corresponding pdf as

$$
b(x)=\frac{d}{d x} B(x)=\frac{T_{a}^{c}(x)}{\overline{T_{a}}} .
$$

By the same token, probability distribution of elapsed idle time may be described by

$$
\begin{aligned}
& A(x)=\frac{1}{\overline{T_{i}}} \int_{0}^{x} T_{i}^{c}(y) d y \\
& a(x)=\frac{d}{d x} A(x)=\frac{T_{i}^{c}(x)}{\overline{T_{i}}}
\end{aligned}
$$

In scenario 1, where the same channel begins and ends the blackout, the PDF of blackout duration can be calculated as

$$
\begin{aligned}
D_{s}(x) & =P(D \leq x \mid \text { scenario } 1) \\
& =T_{a}(x)
\end{aligned}
$$

This scenario occurs if the duration of active channel time (on the channel which started the blackout) is smaller than the duration of residual active channel time, applied to the remaining $N-1$ channels. The probability of this event is

$$
\begin{aligned}
P_{l b} & =\left(\int_{x=0}^{\infty} P\left(T_{a} \leq x\right) P\left(T_{a,-}=x\right)\right)^{N-1} \\
& =\left(\int_{x=0}^{\infty}\left(\int_{y=0}^{x} t_{a}(y) d y\right) b(x) d x\right)^{N-1}
\end{aligned}
$$

Note that for constant values of channel active times, $P_{l b}=0$.

If the channel which began the blackout differs from the one that ends it, as in Fig. 1(b), the PDF of blackout duration $D$ can be obtained as

$$
\begin{aligned}
D_{d}(x) & =P(D \leq x \mid \text { scenario } 2) \\
& =1-(1-B(x))^{N}
\end{aligned}
$$

where $N$ is the number of channels. 
By combining the two scenarios, we obtain the PDF for blackout duration as

$$
D(x)=\left(1-P_{l b}\right) D_{d}(x)+P_{l b} D_{s}(x)
$$

and its pdf as $d(x)=\frac{d D(x)}{d x}$. Then, mean value and variance of blackout duration can be calculated as

$$
\begin{aligned}
\bar{D} & =\int_{x=0}^{\infty} x d(x) d x \\
\operatorname{var}(D) & =\int_{x=0}^{\infty}(x-\bar{D})^{2} d(x) d x
\end{aligned}
$$

We note that expressions (9), (7) and (10) contain linear combinations of PDF-s of products of channel active time and residual active time. This indicates that the variability of blackout duration might be larger than the variability of the corresponding active or residual active time for a single channel. To ascertain this, we need to consider higher moments of the probability distribution or their derivatives or their derivatives, such as the standard deviation, $\sqrt{\operatorname{var}(D)}$, and skewness, defined as

$$
S_{d}=\frac{\left.(-1)^{3} \frac{d^{3} D^{*}(s)}{d s^{3}}\right|_{s=0}-\left.3 \frac{d^{2} D^{*}(s)}{d s^{2}}\right|_{s=0} \bar{D}+2 \bar{D}^{3}}{\operatorname{var}(D)^{3 / 2}}
$$

where $D^{*}(s)=\int_{x=0}^{\infty} e^{-s x} d(x) d x$ denotes the LaplaceStieltjes Transform (LST) for the duration of complete blackout time obtained from the corresponding pdf $d(x)$.

\section{PERformance EVAluation}

To evaluate the impact of primary user activity on the performance of blackouts, we have calculated probabilistic parameters of blackouts. The number of channels in the network was varied between $N=5$ and 50 . We have assumed that primary sources are homogeneous, one per each channel, with activity factor (duty cycle) varied in the range from $p_{\text {on }}=0.1$ to 0.5 .

Using these values, we have conducted three experiments with different distributions of primary user active and idle times; the results of these experiments are shown in Fig. 2. The diagrams in the leftmost column correspond to the case where primary user active and idle times are exponentially (i.e., Erlang-1) distributed random variables, while those in the center column correspond to Erlang-2 distributed active and idle times. Finally, the diagrams in the rightmost column were obtained with constant active and exponentially distibuted idle times, which broadly models the scenario where primary users are 802.11-like nodes [11], [7]. In all three cases, primary users were independent on each channel, and the mean cycle time of each was kept constant at $T_{c y c}=6000$.

The first set of diagrams, shown in top row of Fig. 2, presents the mean value of blackout duration. The shape of all three surfaces is about the same, exhibiting strong dependence on the number of channels and much weaker dependence on the activity factor (duty cycle) of primary sources; however, this dependence becomes more pronounced when the number of channels drops below 15 or so. Exponentially distributed primary user activity leads to the largest mean duration of blackouts, while the mixed distribution with constant active, exponentially distributed idle times leads to shortest mean durations.

Similar results can be observed in case of the standard deviation of blackout duration, shown in the second row of Fig. 2. The differences between the three distributions are more visible here than in the case of mean values, even though the general shape of the surfaces is about the same.

To facilitate comparisons between the three distributions, we have also calculated the coefficient of variation, defined as the ratio of standard deviation and mean value, $c v=\frac{\sqrt{\operatorname{var}(D)}}{\bar{D}}$, which is a proxy measure for the dispersion of values of probability distribution around the mean: the larger it is, the wider the probability distribution is.

From the values shown in the third row of Fig. 2, we can infer that exponential distribution of primary user active and idle leads to hyper-exponential distribution of blackout duration, while the other two distributions (Erlang-2 and constant active, exponentially distributed idle times) result in the coefficient of variation close to the value of 1 . However, results for the former are mildly hyper-exponential, while those for the latter are mildly hypo-exponental.

The fourth, bottommost row of diagrams in Fig. 2 shows the skewness coefficient values for the three distributions. Skewness measures the symmetry of the distribution around the mean value. Values close to zero indicate a symmetric probability distribution, while large positive or negative values indicate that the probability distribution has a long tail to the right or left of the mean, respectively. Large values obtained under exponential and, to a lesser extent, erlang-2 distribution of active and idle times indicate that the blackout distribution has a long tail; in other words, very long blackouts may occasionally occur. The values obtained under constant active, exponentially distributed idle times show a much smaller peak.

In all diagrams, as long as the number of channels is above 15 to 20 , the behavior of all observed variables mean value, standard deviation, coefficient of variation, and skewness - is rather tame and their values are close to the values characteristic for an exponential distribution.

We have also calculated the probability that the duration of a blackout is longer than 100 and 200 time units, assuming (as before) that the mean value of primary activity cycle time is 6000 units. WE have used for the same three distributions of primary user active and idle times used in the previous experiments.

As can be seen from the results shown in Fig. 3, these probabilities decrease rapidly with the increase in the number of channels $N$. While they are higher for higher values of primary user activity factor (duty cycle) $p_{o n}$, even this dependency diminishes when the number of channels increases.

We can also observe that the maximum value is lower for longer blackout duration (as expected), but the difference between values for different distributions of active and idle 


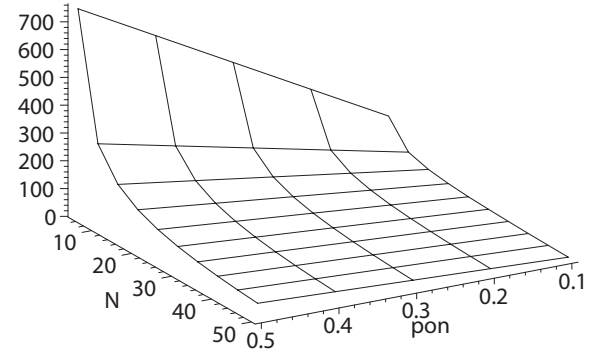

(a) Mean value, under exponentially distributed active and idle times.

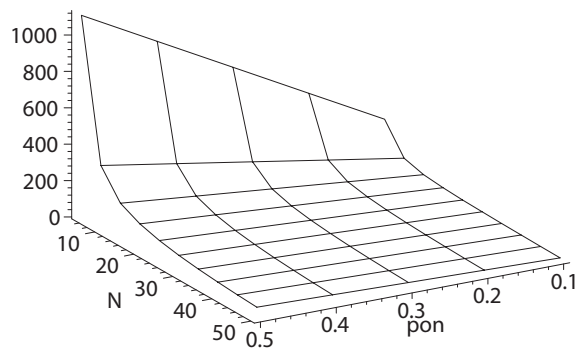

(d) Standard deviation, under exponentially distributed active and idle times.

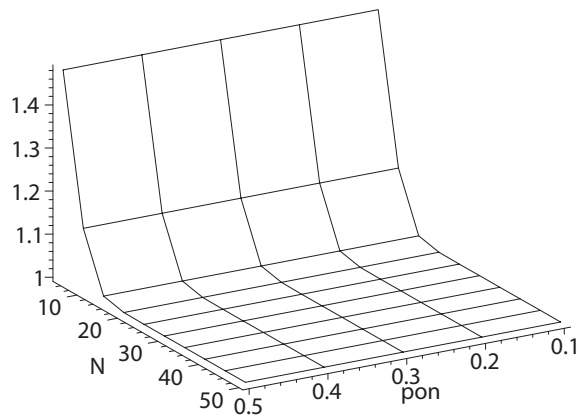

(g) Coefficient of variation, under exponentially distributed active and idle times.

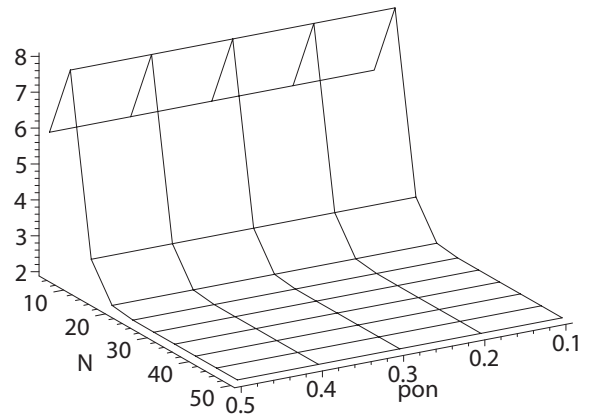

(j) Coefficient of skewness, under exponentially distributed active and idle times.

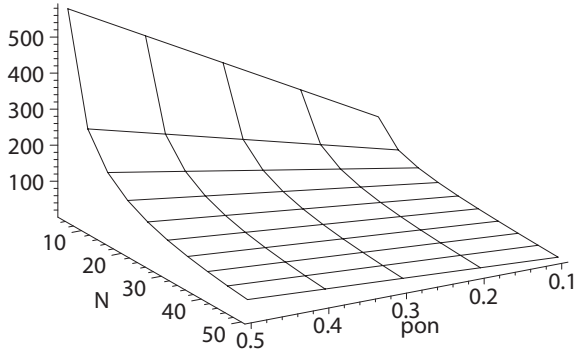

(b) Mean value, under Erlang-2 distribution of active and idle times.

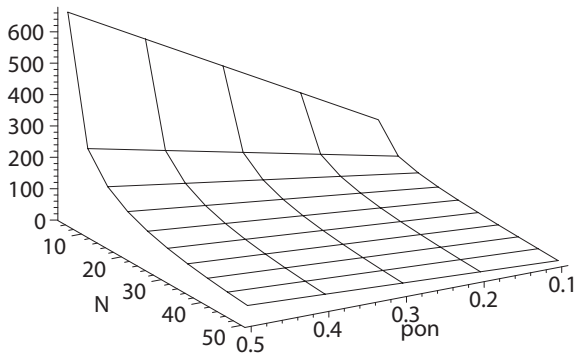

(e) Standard deviation, under Erlang-2 distribution of active and idle times.

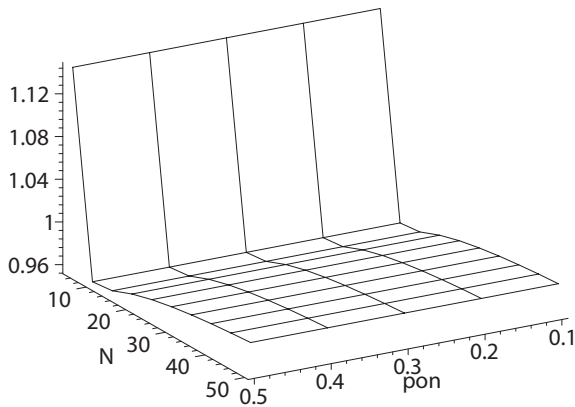

(h) Coefficient of variation, under Erlang-2 distribution of active and idle times.

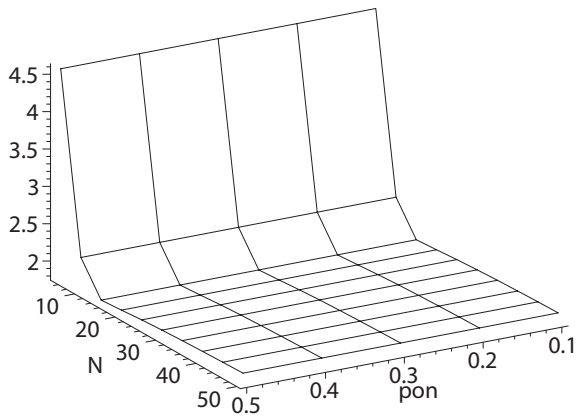

(k) Coefficient of skewness, under Erlang-2 distribution of active and idle times.

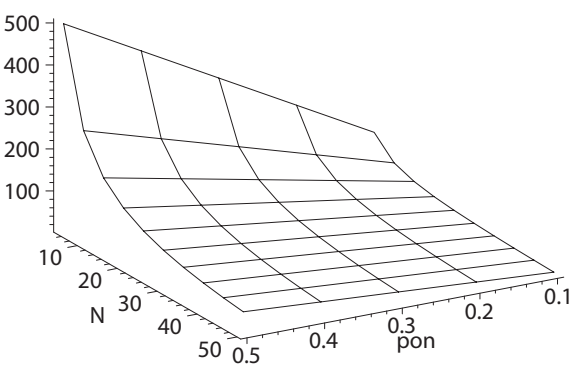

(c) Mean value, under constant active and exponentially distributed idle times.

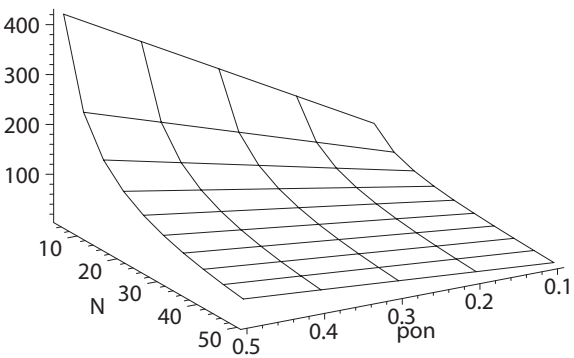

(f) Standard deviation, under constant active and exponentially distributed idle times.

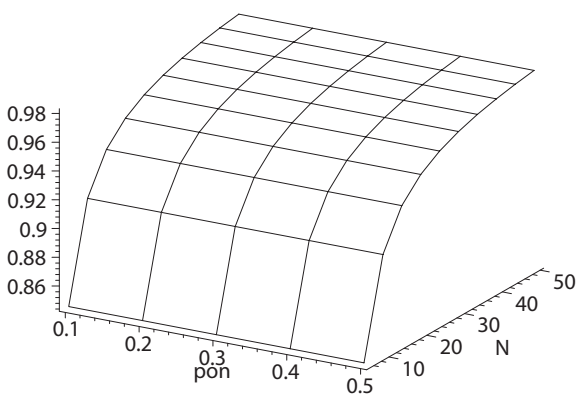

(i) Coefficient of variation, under constant active and exponentially distributed idle times.

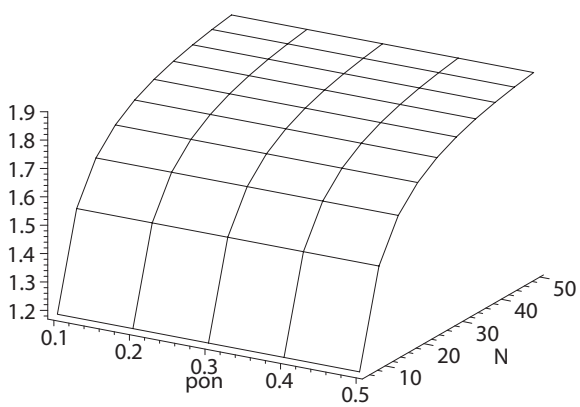

(1) Coefficient of skewness, under constant active and exponentially distributed idle times.

Fig. 2. Blackout duration statistics vs. number of channels and primary user activity factor, under different distributions of active and idle channel times. 


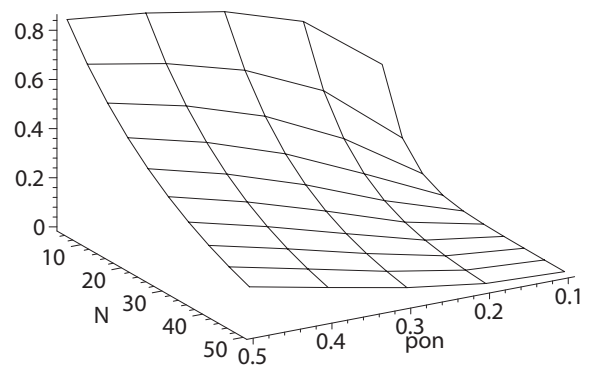

(a) Probability that blackout is longer than 100 slot exponential distribution of active and idle times.

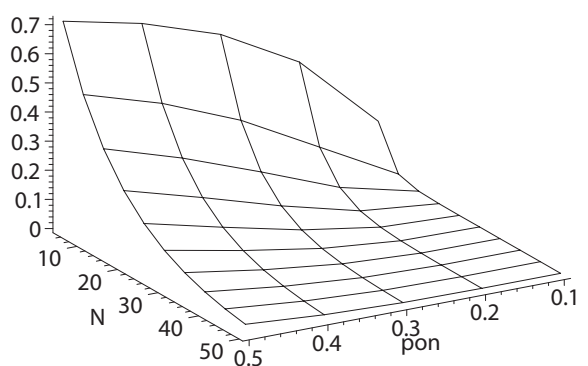

(d) Probability that blackout is longer than 200 slots, exponential distribution of active and idle times.

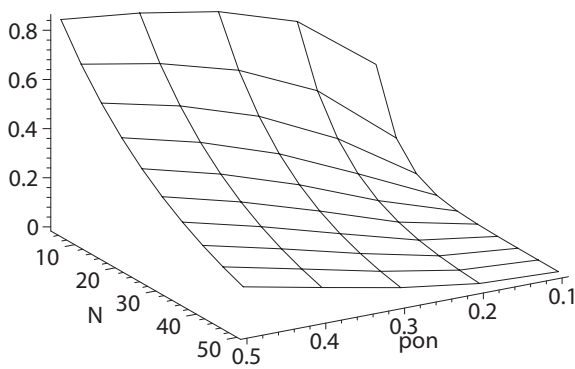

(b) Probability that blackout is longer than 100 slots, Erlang-2 distribution of active and idle times.

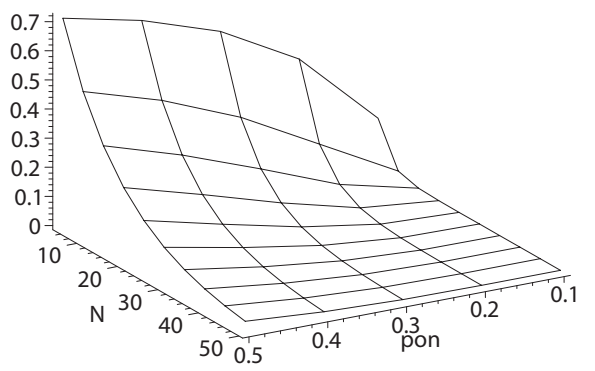

(e) Probability that blackout is longer than 200 slots, Erlang-2 distribution of active and idle times.

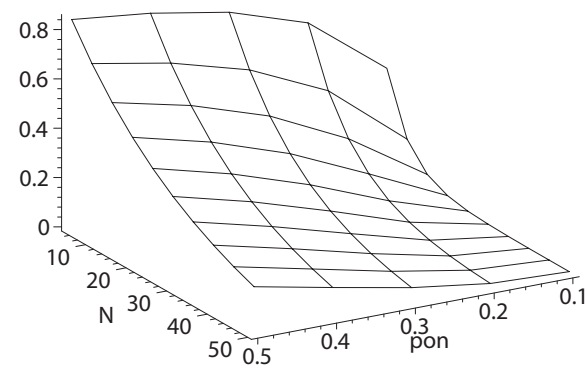

(c) Probability that blackout is longer than 100 slots, constant active and exponentially distributed idle times.

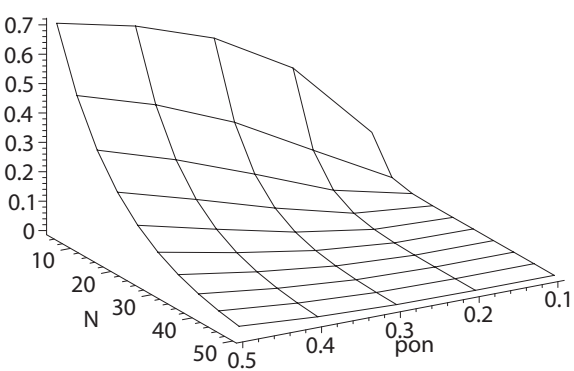

(f) Probability that blackout is longer than 200 slots, constant active and exponentially distributed idle times.

Fig. 3. Probability that blackout time is longer than 100 (top row) and 200 unit time slots (bottom row) under different distributions of primary user active and idle times.

times on the channel (i.e., betwen the diagrams in the same row) are very small, to the point where the surfaces become virtually indistinguishable from one another.

\section{Conclusion}

The presence of primary user activity leads to the creation of blackout periods during which the operation of a cognitive secondary network is impaired or downright impossible. Our analysis confirms this by providing a quantitative insight into the impact of complete blackouts which interrupt the operation of a cognitive network. When the number of channels used by the cognitive network is small - say, below 5 or 10 - the distribution of blackout duration depends very much on the distribution of primary user active and idle times. Moreover, it exhibits a hyper-exponential distribution with a long right tail, since the coefficient of variation is above 1 and the skewness coefficient is above 2 .

However, if the cognitive network uses a larger number of channels such as 15 or more, the duration of total blackouts follows a probability distribution that has coefficient of variation and skewness coefficient that are nearly exponential. Moreover, this effect is nearly independent of the actual distribution of primary user active and idle times.

Our future work will focus on developing MAC protocols that will allow cognitive secondary networks to tolerate blackout periods with minimum interruption, and to allow for quick network formation and recovery.

\section{REFERENCES}

[1] I. F. Akyildiz, W.-Y. Lee, and K. Chowdhury. CRAHNs: Cognitive radio ad hoc networks. Ad Hoc Networks, 7(5):810-836, 2009.

[2] I. F. Akyildiz, W.-Y. Lee, M. C. Vuran, and S. Mohanty. NeXt generation/dynamic spectrum access/cognitive radio wireless networks: A survey. Computer Networks, 50:2127-2159, 2006.

[3] C. Cormio and K. R. Chowdhury. A survey on MAC protocols for cognitive radio networks. Ad Hoc Networks, 7(7):1315-1329, 2009.

[4] D. P. Heyman and M. J. Sobel. Stochastic Models in Operations Research, Volume I: Stochastic Processes and Operating Characteristics. McGraw-Hill, New York, 1982.

[5] P. K. Lee. Joint frequency hopping and adaptive spectrum exploitation In IEEE Military Communications Conference MILCOM2001, volume 1, pages 566-570, Washington, DC, Oct. 2001.

[6] J. Mišić and V. B. Mišić. Simple and efficient MAC for cognitive wireless personal area networks. In Proc. Global Telecommunications Conference GLOBECOM'09, Honolulu, HI, Nov. 2009.

[7] J. Mišić and V. B. Mišić. Characterization of idle periods in IEEE 802.11e networks. In IEEE Wireless Communications and Networking Conference (WCNC 2011, pages 1004-1009, Cancun, Mexico, 2011.

[8] J. Mišić and V. B. Mišić. Recovery using backup channels in channelhopping cognitive networks. In 7th Int. Workshop on Wireless Sensor, Actuator and Robot Networks (WiSARN 2013-Spring), Cambridge, MA, May 2013.

[9] J. Mišić, V. B. Mišić, and M. S. I. Khan. On the selection of working channels in a channel-hopping cognitive PAN. In 9th Int. Wireless Communications and Mobile Computing Conf. (IWCMC 2013), Cagliari, Sardinia, Italy, July 2013.

[10] J. Mišić, H. Khojasteh, N. Khan, and V. B. Mišić. Towards an efficient rendezvous protocol for a cognitive PAN. In IEEE International Conference on Communications (ICC'2012), Ottawa, ON, June 2012.

[11] A. Motamedi and A. Bahai. MAC protocol design for spectrum-agile wireless networks: Stochastic control approach. In 2nd IEEE Int'l Symp. on New Frontiers in Dynamic Spectrum Access Networks DySPAN 2007, pages 448-451, Dublin, Ireland, Apr. 2007. 